\title{
An Analytical Model for Alloyed Ohmic Contacts Using a Trilayer Transmission Line Model
}

\author{
Geoffrey K. Reeves and H. Barry Harrison
}

\begin{abstract}
This paper describes a Transmission Line Model approach to the modeling and analysis of alloyed planar ohmic contacts. It briefly reviews the standard Transmission Line Model (TLM) commonly used to characterize a planar ohmic contact. It is shown that in the case of a typical Au-Ge-Ni alloyed ohmic contact, a more realistic model based on the TLM should take into account the presence of the alloyed layer at the metalsemiconductor interface. In this paper, such a model is described. It is based on three layers and the two interfaces between them, thus forming a Tri-Layer Transmission Line Model (TLTLM). Analytical expressions are derived for the contact resistance $R c$ and the contact end resistance $R e$ of this structure, together with a current division factor, $f$. Values for the contact parameters of this TLTLM model are inferred from experimentally reported values of $R c$ and $R e$ for two types of contact. Using the analytical outcomes of the TLTLM, it is shown that the experimental results obtained using a standard TLM can have considerable discrepancies.
\end{abstract}

\section{INTRODUCTION}

MPROVEMENTS in the reliability and performance of semiconductor devices often require improvements to the quality of their ohmic contacts. The quality of such contacts is measured using various parameters. For the purposes of measuring and comparing the electrical quality of the contact, the specific contact resistance $\rho_{c}$ is probably the most commonly derived and quoted parameter. Thus much of the research on ohmic contacts is devoted to obtaining values for the specific contact resistance and its subsequent minimization. Ohmic contacts are commonly formed by the evaporation of the appropriate metals followed by an alloying or sintering heat cycle in order to bring about an ohmic characteristic to the contact. The measurement of parameters to obtain $\rho_{c}$ enables the influence of the metallizations and the alloying cycle on the contact resistance to be monitored, thus allowing an optimization of the contact procedure.

A variety of metallizations and alloying conditions have been utilized for the formation of ohmic contacts particularly for compound semiconductors [1], [2]. The most studied compound semiconductor ohmic contact is undoubtedly the Au-Ge-Ni alloyed ohmic contact on to n-type GaAs. Electrical measurements on this system generally use the Transmission Line Model (TLM) technique to obtain a value of $\rho_{c}$ for the contact. Additional data on the contact is sometimes reported

Manuscript received October 3, 1994; revised April 13, 1995. The review of this paper was arranged by Associate Editor K. Shenai.

G. K. Reeves is with the Royal Melbourne Institute of Technology, Melbourne, 3001, Australia.

H. B. Harrison is with Griffith University, Nathan, Queensland, 4111, Australia.

IEEE Log Number 9412482. with the derivation of the parameter $R s k$, referred to as the sheet resistance of the semiconducting layer beneath the contact [3].

In addition to the electrical characterization of alloyed ohmic contacts, a considerable amount of research has been undertaken on the physical nature of the Au-Ge-Ni contact in order to understand the mechanisms of the reactions taking place between the metallizations and the underlying GaAs. Vertical cross sections of the alloyed Au-Ge-Ni contact show that following the alloying cycle, a complex alloyed region is formed between the contact metallization and the unreacted GaAs. This interfacial alloyed layer is generally found to have a thickness $t$ of the order of $0.1-0.2 \mu \mathrm{m}$ [3], [4]. In fact from the vertical cross section, the contact structure can reasonably be represented by three distinct layers - the top metallization, the complex alloyed layer (of thickness $t \mu \mathrm{m})$ and the underlying but unreacted GaAs. There thus arises a problem in using the standard TLM for the electrical characterization of such a contact as the standard Transmission Line approach models the contact as a two layer structure-the metallization and the underlying semiconductor, with one interface between the two layers. A more realistic electrical model should acknowledge the existence of the alloyed layer.

Various modifications to the standard TLM have been developed in order to more closely model the particular contact system being characterized. Thus an extended TLM was proposed [5] to take into account an additional voltage drop in the semiconductor layer in the contact region, modified TLM's were developed to account for the finite sheet resistivity of the two contacting layers [6], [7], an additional interface was added to account for the AlGaAs layer when modeling HEMT contacts [8] and the current flow in the active layer of MOSFET's was divided into two regions of current flow at fixed depths below the contact [9].

However in the case of alloyed contacts, a more flexible and comprehensive model is needed to accurately represent the observed physical characteristics of this contact ${ }^{1}$. A Tri-layer Transmission Line Model (TLTLM) is presented here in order to electrically represent the metal layer, the alloyed region layer and the unreacted semiconductor layer that together make up the contact region. The model allows the alloyed contact layer and the unreacted semiconductor layer to have separate and identifiable sheet resistances, while the top conducting layer is assumed to have zero sheet resistance. It also allows

\footnotetext{
'Shur has briefly discussed the desirability of having a more comprehensive model for characterizing alloyed ohmic contacts in GaAs Devices and Circuits, M. Shur, Plenum Press, NY, 1987, p. 156.
} 
two interfaces--one between the metal and alloyed layer and the second between the alloyed and semiconductor layer-to be individually represented. The effects of current crowding at the leading edge of the contact and its effect on contact current flow and on the contact resistance can also be evaluated.

In Section II of this paper a brief review of the standard TLM is given. This model is commonly used to measure the specific contact resistance of planar ohmic contacts including alloyed ohmic contacts to semiconductors such as GaAs. In Section III, the TLTLM is described in detail and the equations for contact current flow, current division, the contact resistance $R c$ and the contact end resistance $R e$ are given. In Section IV, the effects of the various contact parameters on the current flow beneath the contact and the calculated contact resistance are discussed. The detailed derivation of the equations given in Section III is provided in an appendix.

\section{STANDARD TLM NETWORK}

The specific contact resistance is a parameter that allows an easy quantitative comparison to be made between contacts independent of their geometry. A wide variety of test structures have been designed in order to provide measurements that extract the specific contact resistance $\rho_{c}$ of metal-semiconductor ohmic contacts [5], [10]-[13]. (The specific contact resistance is a normalized value of the contact resistance making it independent of the area of the contact and allowing easy calculation of contact resistances for contacts with differing areas). A brief description of the most commonly used test structure patterns is provided in [14].

It is important to clarify exactly what is being obtained when the contact resistance $R c$ is measured from the various test structures. The value of $R c$ depends on the type of contact and thus the nature of the current flow in the contact region. The two contact geometries commonly encountered are the planar and the "sandwich" contact which have quite different current flow patterns. In a "sandwich" contact the current flow is normal and uniform in current density to the plane of the contact. The derivation of the specific contact resistance $\rho_{c}$ from the contact resistance is quite straight forward as $\rho_{c}=R c \cdot A$ where $A$ is the area of the contact with $\rho_{c}$ usually measured in $\Omega \cdot \mathrm{cm}^{2}$.

For the planar contacts considered in this paper, the contact resistance $R c$ is taken as the resistance current flow encounters after passing under the leading edge of the contact as shown in Fig. 1(a). Fig. 1(b) displays the nonuniform current flow encountered beneath the planar contact. The electrical network for the planar contact uses a one-dimensional model for the current flow where the flow is taken parallel to the plane of the contact. The standard TLM [5], [10] electrically models the planar contact as a network of distributed resistive elements as illustrated in Fig. 2. Rsh is the semiconductor sheet resistance $(\Omega / \square)$ outside the contact area while the resistance $R s k$ represents the sheet resistance $(\Omega / \square)$ of the semiconductor layer beneath the contact (a different element value is used as this sheet resistance may differ from $R s h$ ). The resistive element $\rho_{c}$ represents the narrow carrier depleted region occurring at the metal-semiconductor interface. Hence

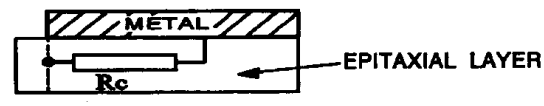

(a)

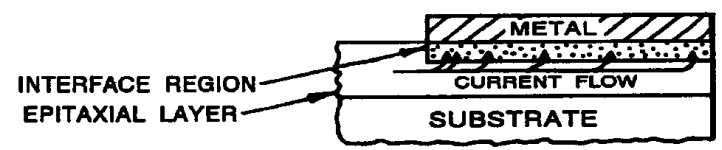

(b)

Fig. 1. (a) $R c$ boundary and (b) current flow for a planar ohmic contact.

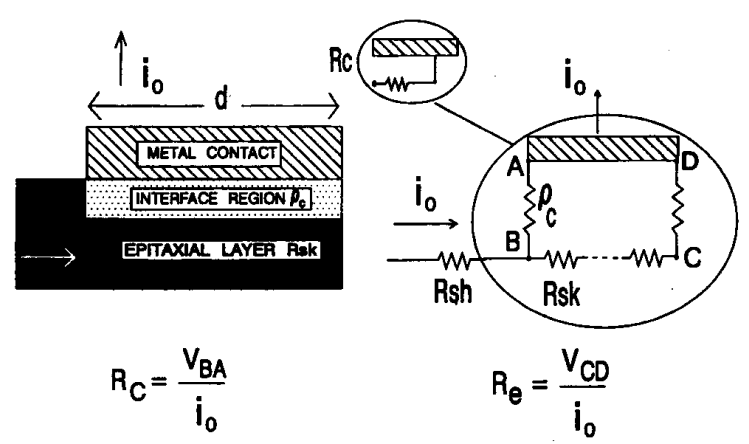

Fig. 2. Planar ohmic contact and standard TLM electrical network representation.

the voltage drop across this region is caused by the presence of $\rho_{c}$. The voltage drop beneath the contact in the horizontal direction is attributed solely to the current flow in $R s k$ while the voltage drop in the vertical direction (perpendicular to the plane of the contact) is due to $\rho_{c}$. The contact has a length $d$ and a width $w$.

With reference to Fig. 2, $R c$ and $R e$ are defined as

$$
R c=V_{B A} / i_{0} \text { and } R e=V_{C D} / i_{0} .
$$

From these definitions, it may be shown [5] that the following relationships hold for the contact modeled in Fig. 2

$$
\begin{aligned}
R e & =\left[\left(R s k \cdot \rho_{c}\right)^{1 / 2} / w\right] / \sinh (\alpha d) \quad \text { and } \\
R c & =\left[\left(R s k \cdot \rho_{c}\right)^{1 / 2} / w\right] \cdot \operatorname{coth}(\alpha d)
\end{aligned}
$$

where

$$
\alpha=\left(R s k / \rho_{c}\right)^{1 / 2} .
$$

$R c$ and $R e$ are experimentally determined using a standard TLM test pattern consisting of three or more identical contacts on an electrically isolated semiconductor region. The test pattern also allows the determination of $R s h$ [12]. With $R c$ and $R e$ known, the values of $\rho_{c}$ and $R s k$ may be found from (2). If it can be safely assumed that for a given contact, $R s k=R s h$ (which only occurs in certain cases), then only a measurement of $R c$ is required in order to find $\rho_{c}$. Note that the quantity $\alpha$ is often replaced by the parameter $L_{t}$ where $L_{t}=1 / \alpha$, and is termed the contact transfer length-a useful concept introduced by Shockley [10]. With reference to the standard TLM, and where the contact length $d \gg L_{t}$, the current flow 


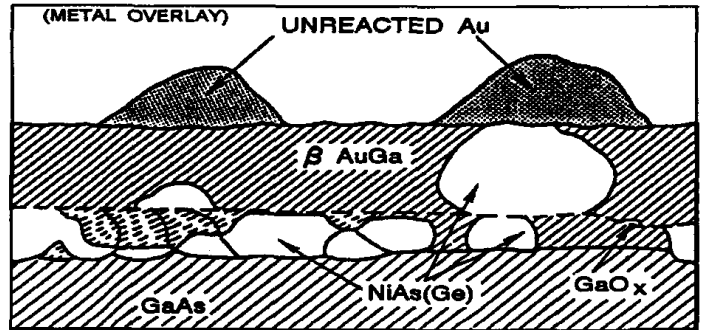

Fig. 3. Contact microstructure for an alloyed Au-Ge-Ni contact [18].

decreases close to exponentially in the semiconductor layer. Thus the transfer length $L_{t}$ is the distance along the contact from the leading edge for the current in the semiconducting layer to fall to $1 / e$ of its initial value as it transfers to the metal layer.

\section{THE TLTLM NETWORK}

Ohmic contacts to n-type GaAs are commonly made by evaporating a combination of $\mathrm{Au}-\mathrm{Ge}-\mathrm{Ni}$ followed by an alloying cycle. This alloying cycle causes $\mathrm{Ga}$ from the $\mathrm{GaAs}$ to outdiffuse into the contact metallization, creating $\mathrm{Ga}$ vacancies which can then be occupied by the Ge. The Ge thus acts as a donor resulting in a highly doped surface region (typically $>10^{18} \mathrm{~cm}^{-3}$ ) just below the contact metal. This highly doped region narrows the metal semiconductor barrier allowing tunnelling of carriers to take place. Although this explains in a simple way the observed ohmic contact behavior, a much more complex process is known to take place. Many detailed studies have been performed on this contact system [4], [15]-[17]. The alloyed layer at the metal-semiconductor interface is generally observed to have the physical microstructure as shown in Fig. 3 [18]. When taken on a microstructure scale, this layer is clearly not one of uniform composition and is more akin to a matrix of various compounds and/or alloys. Thus on a microscale, the concept of a uniform sheet resistance for the alloyed layer is not appropriate. However, when scaled over an area the size of a typical ohmic contact, an average resistivity (and thus sheet resistance) can be ascribed to this layer. (All ohmic contact test patterns which provide data on specific contact resistance and sheet resistance give this data as an average over a given contact area). Provided device contact sizes and the sizes of contact test structures significantly exceed the size of the alloyed layer inhomogeneities, then the concept of a sheet resistance remains a viable one for the alloyed layer.

In the case of a Au-Ge-Ni contact on GaAs, the alloyed layer is found to consist of $\mathrm{Ni}-\mathrm{Ge}-\mathrm{As}$ compounds in a matrix of $(\mathrm{Au}, \mathrm{Ga})$ alloys. The alloyed ohmic contact clearly consists of more than just a metal on an epitaxial layer of semiconductor. In fact the contact structure can reasonably be represented by three distinct layers - the top metallization, the alloyed contact layer and the underlying but unreacted epitaxial GaAs. On sectioning the contact, the alloyed layer is generally found to have a thickness $t$ of the order of $0.1-0.2 \mu \mathrm{m}$ [3], [4]. Even the alloying of hypoeutectic Au-Ge-Ni compositions can result in an alloyed layer depth of this order [19]. This depth can represent a significant proportion of the epilayer thickness

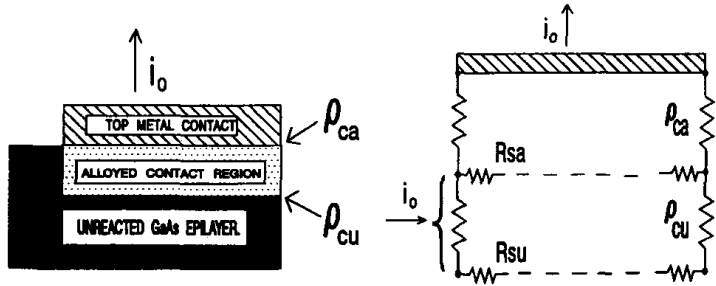

$$
\begin{aligned}
& \text { metal - alloyed semicond. interface } P_{\text {ca }} \text { alloyed semiconductor layer Risa } \\
& \text { ALOYED-UNALLOYED SEMICOND. INTERFACE } P_{\text {cU }} \text { UNALLOYED SEMICONDUCTOR LAYER RSU }
\end{aligned}
$$

Fig. 4. Alloyed ohmic contact cross section and its corresponding TLTLM electrical network representation.

of FET devices. As a result of the alloying process, the combined sheet resistance of the semiconductor-alloy layers under the contact will probably have altered. This change can be represented in the standard TLM by using $R s k$ to represent the magnitude of the sheet resistance of this region, as opposed to assuming that the underlying semiconductor region of the contact still retains its unalloyed value of $R s h$. However the use of a single modified resistance to represent the alloyed layer, the semiconducting layer and the interface between them is unsatisfactory. Physically, no single resistive layer of $R s k$ exists under the contact and there is an additional voltage drop in the vertical direction due to the interface. This additional voltage drop cannot be represented by $R s k$.

This deficiency can be overcome by using a Tri-Layer TLM (TLTLM) in order to electrically model the properties of the alloyed layer. Fig. 4 shows the cross section of the alloyed contact and its corresponding TLTLM electrical network. The voltage drop at the metal to alloyed layer interfacial barrier occurs across the specific contact resistance element $\rho_{c a}$, while the voltage drop across the alloyed layer to the unreacted GaAs layer occurs across $\rho_{c u}$, the specific contact resistance at this interface. The sheet resistance of the metal layer is taken as zero, while the alloyed layer and the underlying epilayer have sheet resistances $R s a$ and $R s u$ respectively. The currents flowing in $R s u$ and $R s a$ are taken as $i_{1}$ and $i_{2}$ respectively. As noted previously in this section, the alloyed layer is not homogeneous on the microstructure scale. Thus in a contact system where the contact process has resulted in such inhomogeneities, there will naturally be corresponding regions where the current $i_{2}$ will have local fluctuations from its average value.

The complete TLTLM network is shown in Fig. 5. Current $i_{3}$ is the current collected by the metal layer of the contact between $x=0$ (leading edge of the contact) and the point $x$ back from the leading edge. Thus the total contact current $i_{0}=i_{1}(x)+i_{2}(x)+i_{3}(x)$, and at $x=d i_{1}(d)$ and $i_{2}(d)=0$ and $i_{3}(d)=i_{0}$. Fig. 5 also includes a contact front edge resistor $R f$ which accounts for the fraction of current entering the sidewall of the alloyed layer. Since the alloyed layer has a depth $t$ which may be a significant fraction of the total epilayer depth, it is clear that a proportion of the contact current will enter the contact region via this sidewall. Of the total contact 


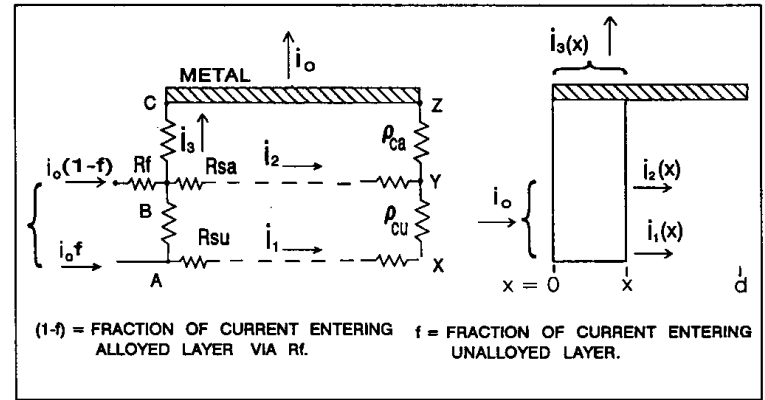

Fig. 5. The complete TLTLM network showing front edge resistance $R f$ and current components $i_{0} f$ and $i_{0}(1-f)$ entering the contact layers.

current, $i_{0}$, a fraction $(1-f)$ is taken as entering the contact through the alloyed layer sidewall while the remainder $f$, enters in the conventional TLM manner-via Rsu under the contact. Since a portion of the current enters the sidewall of the alloyed layer (width $w$ and thickness $t$ ), then assuming a uniform current density across the area (w.t) of this sidewall, the magnitude of $R f$ for the contact is given by

$$
R f=\rho_{c u} / \text { (w.t). }
$$

Referring to Fig. 5, $R c$ and $R e$ are defined in a similar manner as for the standard TLM

$$
R c=V_{A C} / i_{0} \text { and } R e=V_{X Z} / i_{0} .
$$

The two entry points at the leading edge of the contact for the currents $i_{0} f$ and $i_{0}(1-f)$ are taken as being at the same potential. The network of resistive elements in Fig. 5 may be solved to give the current flows $i_{1}, i_{2}$ and $i_{3}$ as well as calculated values of $R c, R e$ and the current division factor, $f$. The method used for this derivation is outlined in the appendix. The following equations are thus obtained for $R c, R e$ and $f$ (see also (8) at the bottom of this page):

$$
\begin{aligned}
R c= & K \cdot\left\{\frac{f \cdot\left(R s u-\rho_{c u} \cdot a^{2}\right)-(1-f) \cdot R s a}{b \cdot \tanh (b d)}\right. \\
& \left.-\frac{f \cdot\left(R s u-\rho_{c u} \cdot b^{2}\right)-(1-f) \cdot R s a}{a \cdot \tanh (a d)}\right\} \\
R e= & K \cdot\left\{\frac{f \cdot\left(R s u-\rho_{c u} \cdot a^{2}\right)-(1-f) \cdot R s a}{b \cdot \sinh (b d)}\right. \\
& \left.-\frac{f \cdot\left(R s u-\rho_{c u} \cdot b^{2}-(1-f) \cdot R s a\right.}{a \cdot \sinh (a d)}\right\}
\end{aligned}
$$

where

$$
\begin{aligned}
a & =\sqrt{\left[\left\{c-\left(c^{2}-4 \cdot R s u \cdot R s a /\left(\rho_{c u} \cdot \rho_{c a}\right)\right)^{1 / 2}\right\} / 2\right]} \\
b & =\sqrt{\left[\left\{c+\left(c^{2}-4 \cdot R s u \cdot R s a /\left(\rho_{c u} \cdot \rho_{c a}\right)\right)^{1 / 2}\right\} / 2\right]} \\
c & =\left[(R s a+R s u) / \rho_{c u}+R s a / \rho_{c a}\right] \\
K & =R s u /\left[\rho_{c u} \cdot w \cdot\left(b^{2}-a^{2}\right)\right] \text { and } \\
N & =R f \cdot w \cdot\left(b^{2}-a^{2}\right) \cdot \tanh (b d) \cdot \tanh (a d) .
\end{aligned}
$$

The equations for $R c$ and $R e$ reduce to the corresponding standard TLM (2) and (3) when all the contact current enters the $R s u$ layer (take $f=1$ ) and the presence of the alloyed layer $R s a$ is ignored $(R s a \rightarrow \infty)$. Thus

$$
\begin{aligned}
& R e=\left[\left(R_{s u} \cdot\left(\rho_{c a}+\rho_{c u}\right)\right)^{1 / 2} / w\right] / \sinh (\alpha d) \text { and } \\
& R c=\left[\left(R_{s u} \cdot\left(\rho_{c a}+\rho_{c u}\right)\right)^{1 / 2} / w\right] \cdot \operatorname{coth}(\alpha d)
\end{aligned}
$$

where

$$
\alpha=\left(R_{s u} /\left(\rho_{c a}+\rho_{c u}\right)\right)^{1 / 2} .
$$

For these conditions the network of Fig. 5 thus has the quantity $\left(\rho_{c a}+\rho_{c u}\right)$ representing $\rho_{c}$ and $R s u$ representing Rsk.

\section{DISCUSSION}

\section{A. Current Division $f$}

Equations (A11) and (A12) for $i_{1}(x)$ and $i_{2}(x)$, as derived in the appendix, may be plotted to illustrate the way in which the currents in $R s u$ and $R s a$ are distributed along the contact length. For a contact of $d=10 \mu \mathrm{m}$ and selecting values of $R s a$ and $R s u$ of 100 and $500 \Omega / \square$, respectively, and $\rho_{c a}$ and $\rho_{\mathrm{cu}}$ values of 1 and $10 \times 10^{-6} \Omega \cdot \mathrm{cm}^{2}$, then Fig. 6(a) shows the current distributions for $f=1.0$ and Fig. 6(b) shows the distribution for $f=0.25$. Note that the actual value of $f$ for a given contact is determined by the contact parameters as defined in (8). These same contact parameters are used in an example to illustrate the influence that $R f$ has on the current division factor. Since $\rho_{c u}$ is kept constant at $10 \times 10^{-6} \Omega \cdot \mathrm{cm}^{2}$ in this example, the variation in $R f$ can be taken as being due to a variation in the depth $t$ of the alloyed layer (4). The dependency of $f$ on $R f$ (or $t$ ) and on the contact length is shown in Fig. 7 where $f$ is plotted as a function of the contact length for various values of $R f$. The corresponding values of $t$ (for a contact width $w=100 \mu \mathrm{m}$ ) are shown in parentheses. Note that for the same $R f$ value, if $\rho_{c u}$ decreases by a factor of 10 , the values of $t$ also decrease by 10 . Only for very short contacts does $f$ vary with the contact length.

\section{B. Transfer Length $L_{t}$}

The concept of contact transfer length for planar ohmic contacts was put forward by Shockley [10]. With reference to the standard TLM, and where the contact length $d \gg L_{t}$, the current flow decreases exponentially in the semiconductor layer. Thus the transfer length $L_{t}$ is the distance of the contact traversed in order for the current in the semiconducting layer to fall to $1 / e$ of its initial value as it transfers to the metal layer. In the standard TLM $L_{t}$ is given by (3). However it should be noted that in deriving the equation for $L_{t}$ an exponential current decay below the contact is assumed. For a TLTLM network contact, the current distribution of $\left(i_{1}+i_{2}\right)$ is not

$$
f=\left\{\frac{N+R s a \cdot[b \cdot \tanh (a d)-a \cdot \tanh (b d)]}{N+\left(R s a+R s u-\rho_{c u} \cdot a^{2}\right) \cdot b \cdot \tanh (a d)-\left(R s a+R s u-\rho_{c u} b^{2}\right) \cdot a \cdot \tanh (b d)}\right\}
$$




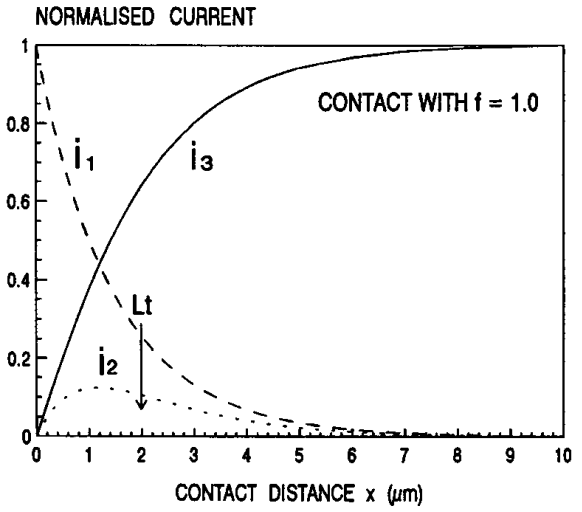

(a)

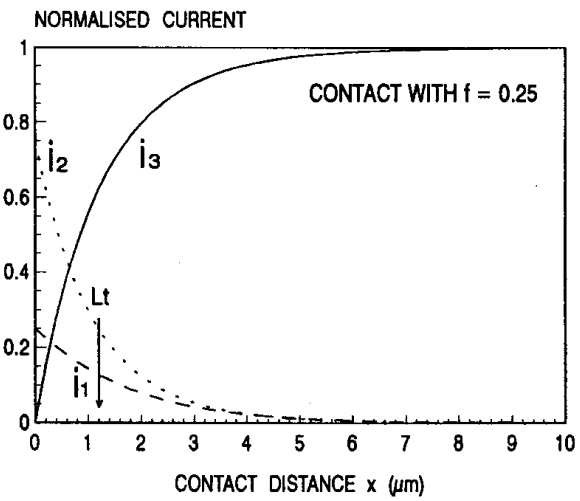

(b)

Fig. 6. Current distribution within Rsa $\left(i_{2}(x)\right)$ and $R s u\left(i_{1}(x)\right)$ as a function of contact distance for a $10 \mu \mathrm{m}$ contact where (a) $f=1.0$ and (b) $f=0.25$.

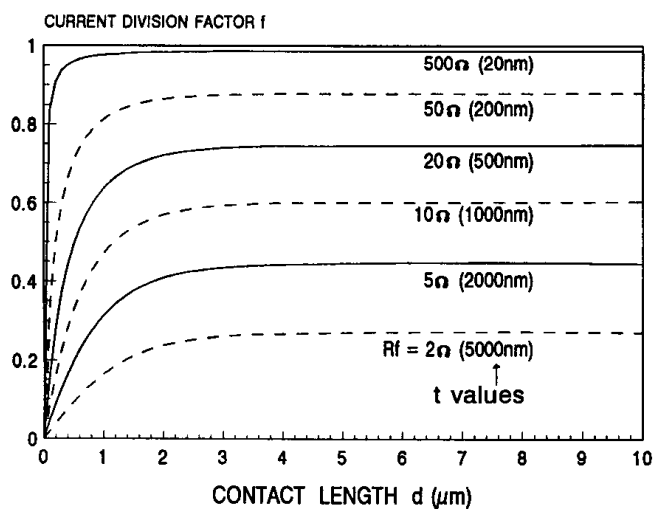

Fig. 7. Current division factor $f$ as a function of contact length for various $R f$ values. Corresponding values of $t$ are shown in parentheses for $w=100 \mu \mathrm{m}$ and $\rho_{c u}=10^{-5} \Omega \cdot \mathrm{cm}^{2}$.

exponential even for a long contact, and thus the use of (3) is not appropriate. For a contact described using the TLTLM network, a more accurate representation of the transfer length $L_{t}$ can be found from a graph of the current distribution in the contact. By taking a contact where $d$ is $\gg L_{t}$, then $L_{t}$ can be defined as the distance taken for the combined currents $i_{1}$ and $i_{2}$ to fall to $1 / e$ of their initial value (or for $i_{3}$ to reach $63 \%$ of its final value). For example, from the current distribution given in Fig. 6(a) and (b), the respective transfer lengths for this contact are $2.0 \mu \mathrm{m}$ and $1.2 \mu \mathrm{m}$. Other examples of $L_{t}$ values for contacts are given in Sections IV-C and IV-D.

\section{Analysis Using the TLTLM}

Analysis using the standard TLM generally involves the experimental determination of $R c$ and $R e$, and then applying the standard TLM (2) and (3), to calculate unique values for $\rho_{c}$ and $R s k$. However the standard TLM test pattern does not provide sufficient data for a unique determination of the TLTLM parameters $\left(\rho_{c a}, \rho_{c u}, R s a\right.$ and $\left.R s u\right)$. Despite this, a good indication of the magnitude of these parameters may be found by using (6) and (7). A set of possible TLTLM parameters is chosen by noting that (6) and (7) must be satisfied as well as considering TLTLM values which are consistent with the semiconductor material from which the test pattern is fabricated. This particularly applies to the sheet resistance $R s a$ and $R s u$. For example, a reasonable estimate for $R s u$ can be obtained from the sheet resistance $R s h$ of the active layer of the test pattern structure. The value of $R s u \geq R s h$ as some of the original epitaxial layer (sheet resistance $R s h$ ) is consumed in the alloying process, leaving a thinner unreacted epitaxial layer (sheet resistance $R s u$ ) beneath the contact. The size of the difference is dependent on the value of $t$ resulting from alloying the contact; as previously noted values of $\sim 0.1-0.2 \mu \mathrm{m}$ are commonly observed. Thus an alloyed ohmic contact with $t=0.1 \mu \mathrm{m}$ on a uniformly doped active layer of $0.3 \mu \mathrm{m}$ thickness, would result in a $R s u$ of $3 / 2 \times R s h$. The possible range for $R s a$ is also a function of the alloying process. By taking $t=0.1 \mu \mathrm{m}$, and assuming that the alloying process in GaAs produces a highly doped alloyed layer beneath the contact, then $R s a$ could reasonably fall in the range of $10-100 \Omega / \mathrm{sq}$. The range of values for $\rho_{c a}$ and $\rho_{c u}$ will be of the same order as $q_{c}$, the specific contact resistance derived using the standard TLM analysis. The magnitude of $R f$ depends primarily on $\rho_{c u}$ (4), with some further variation occurring if the depth, $t$, of the alloyed layer varies.

An example of assigning TLTLM parameters to an alloyed GaAs ohmic contact is shown in Table I where Henry's [20] result for his sample 5-B/8.6 is used. (Henry reported values for $t$ of $0.1-0.125 \mu \mathrm{m}$ for his alloyed contacts). For this test pattern $(w=100 \mu \mathrm{m})$, the $R c$ and $R e$ values were measured as $1.6 \Omega$ and $0.144 \Omega$, respectively, giving $\rho_{c}=$ $2.6 \times 10^{-6} \Omega \cdot \mathrm{cm}^{2}$ and $R s k=100 \Omega / \mathrm{sq}$. using the standard TLM analysis. Rsh was measured as $284 \Omega /$ sq. While two possible sets of TLTLM parameters are given in Table I, the possible set values are constrained by the requirement to match the observed values of $R c$ and $R e$ and by the restrictions placed on Rsa as previously noted. The range of $R s u$ is $3 / 2 \times R s h$ as the implant depth was $0.35 \mu \mathrm{m}$ [20]. $R f$ is calculated from (4) using the corresponding $\rho_{c u}$ value in Table I, while the range for $R s u$ corresponds to a $40-55 \%$ increase in $R s h$, due to the decreased active layer thickness beneath the contact. Table I also shows that for this contact, a significant 
TABLE I

TlTLM Contact Parameters for Au-Ge-Ni OHMIC Contact

\begin{tabular}{|cccccc|cc|}
\hline \multicolumn{7}{|c|}{ Experimental values for Rc, Re and $\rho_{\mathrm{c}}: 1.6 n, 0.144 \Omega$ and $2.6 \times 10^{-6} \mathrm{\Omega .cm} \mathrm{cm}^{2}$} \\
\hline \multicolumn{7}{|c|}{ TLTLM Contact Parameters: } & \multicolumn{2}{c|}{ Calculated Rc and Re values } \\
\hline Rf & Rsu & Rsa & $\rho_{\mathrm{ca}}$ & $\rho_{\mathrm{cu}}$ & $(1-\mathrm{f})$ & $\mathrm{Rc}$ & $\mathrm{Re}$ \\
\hline 4 & 440 & 34.4 & 1.5 & 0.4 & 0.24 & 1.6 & 0.144 \\
5 & 400 & 25.6 & 1.34 & 0.5 & 0.21 & 1.6 & 0.144 \\
\hline
\end{tabular}

Resistance values in $\mathrm{\Omega}$, sheet resistance in $\Omega / \mathrm{sq}$ and $\rho_{\mathrm{c}}$ values are $\times 10^{-6} \mathrm{\Omega} . \mathrm{cm}^{2}$

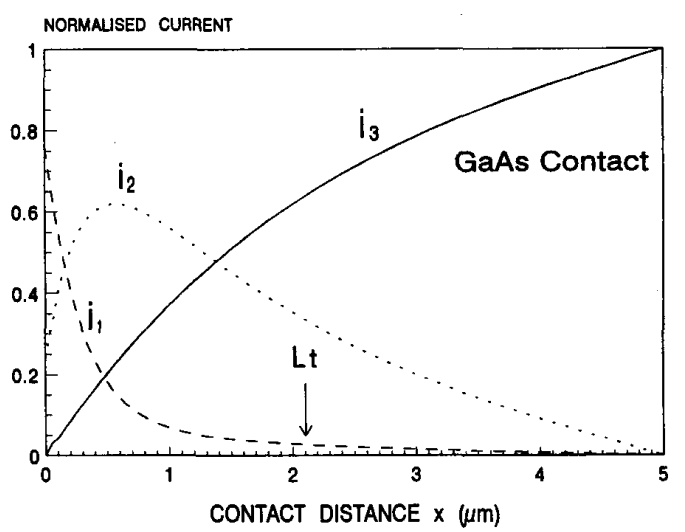

Fig. 8. Current distribution within the contact layers as a function of contact distance traversed in a GaAs Au-Ge-Ni alloyed contact. $L_{t}$ marks the value of the contact transfer length.

part $(1-f)$ of the contact current enters the sidewall of the alloyed layer. The calculated $R c$ and $R e$ values in Table I are the values calculated using the TLTLM parameters listed and (6) and (7).

The contact current distributions $i_{1}, i_{2}$ and $i_{3}$ for the first set of values in Table I are shown in Fig. 8 as a function of the distance traversed beneath the contact, where the total contact current $i_{0}$ is taken as unity. The contact transfer length $L_{t}$ can be derived from Fig. 8. The value of $L_{t}$ corresponds to the contact distance traversed in order for $63 \%$ of the device current to have transferred to the metal layer. From Fig. 8 this occurs at $2.1 \mu \mathrm{m}$. Note that this value is somewhat longer than the transfer length of $1.6 \mu \mathrm{m}$ which is derived using the standard TLM equation (3).

\section{Other Contact Structures}

Although the analysis and discussion just given refer to alloyed ohmic contacts on GaAs, the TLTLM can be applied to other contact structures. The $n^{+} / n$ structure is one example, where a highly doped contact layer is grown on an n-type epitaxial layer. This technique allows a non alloyed ohmic contact to be formed [21], [22], as the metal $\mathrm{n}^{+}$barrier is narrow enough to allow the charge carriers to readily tunnel through it. A detailed analysis of the current transport mechanisms has been given [23] for the non-alloyed $n^{+}$. $\mathrm{n}$ contact, including a derivation of $\rho_{c}$ for an ohmic $\mathrm{Al}$ $\mathrm{n}^{+} \mathrm{GaAs}$ to GaAs contact. The contact geometry here is of the

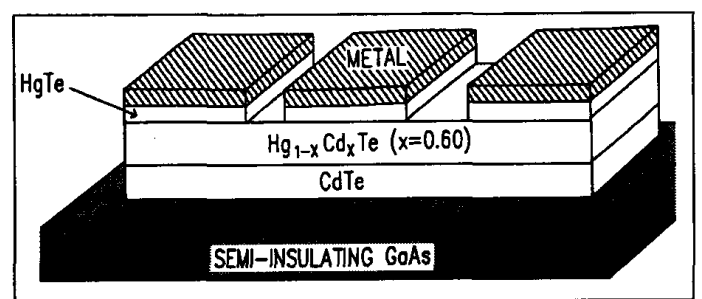

Fig. 9. Cross section of the $\mathrm{Au}-\mathrm{In} / \mathrm{HgTe} / \mathrm{HgCdTe}$ heterojunction contact pattern test structure [24].

"sandwich" type described in Section II and the subsequent analysis thus differs from the planar contacts considered here.

A similar structure occurs in some nonalloyed heterojunction contacts, where a thin layer of a different (low bandgap) semiconductor material is deposited on the epitaxial device layer. The heterojunction approach is particularly applicable to compound semiconductors where narrow bandgap lattice matched materials can be epitaxially grown on wider bandgap device material. One example is the use of a $\mathrm{HgTe}$ layer to contact the wider bandgap $\mathrm{Hg}_{1-x} \mathrm{Cd}_{x} \mathrm{Te}$ (MCT) material [24]. A cross section of the TLM test patterns used to measure $R c$ and $R e$ is shown in Fig. 9. In one test pattern, $R c$ was $88.1 \Omega, R e=0.75 \Omega$ resulting in $R s k=481 \Omega /$ sq. and $\rho_{c}=1.46 \times 10^{-3} \Omega \cdot \mathrm{cm}^{2}$. Applying the TLTLM to this structure, Rsa becomes the sheet resistance of the HgTe layer $(0.2 \mu \mathrm{m}$ thick) and $R s u$ that of the MCT layer. Since the HgTe is etched away outside the contact region there will be no current entering the sidewall of the HgTe layer (equivalent to the alloyed layer in the alloyed contact). Thus in using the TLTLM to analyse this structure, $R f$ will be infinite (i.e., $f=1.0$ ). (Unlike the alloyed contact, it is possible for nonalloyed $\mathrm{n}^{+} / \mathrm{n}$ and heterojunction contacts to have $R f \rightarrow 0$ when the $\mathrm{n}^{+}$or the HgTe layer (see Fig. 9) has not etched away between the contacts). The sheet resistance of the $\mathrm{HgTe}$ layer was measured as $15-16 \Omega / \mathrm{sq}$ while the test pattern of Fig. 9 gave $R s h$ for the MCT layer $\sim 500 \Omega /$ sq. Since the MCT layer is the same outside the contact as under it, the value of $R s u$ is taken as $500 \Omega$ /sq. The current distribution for this contact is illustrated in Fig. 10. Compared to the AuGe-Ni contact current distribution of Fig. 8, the current $i_{2}$ in the $R s a$ layer is somewhat less due largely to the fact that there is no sidewall entry. The value of $L_{t}$ for the heterojunction contact is found from Fig. 10 to be $26.3 \mu \mathrm{m}$. Table II gives two sets of TLTLM parameters that have been derived for this contact in order to match the experimental values of $R c$ and $R e$, and also taking into account small variations in $R s u$.

A point of interest with this contact is the fact that despite all the contact current entering $R s u(f=1.0)$, some horizontal current flow within the Rsa layer still occurs. Hence the sheet resistance of this layer will still affect the contact properties. However the magnitude and distribution of the current in the $R s a$ layer is much reduced for this contact when compared to the alloyed Au-Ge-Ni contact. Thus the influence of the magnitude of Rsa on the contact and in particular on the value of $R s k$ is considerably less than for the Au-Ge-Ni contact. Hence $R s k$ for the heterojunction contact $481(\Omega / s q)$ 


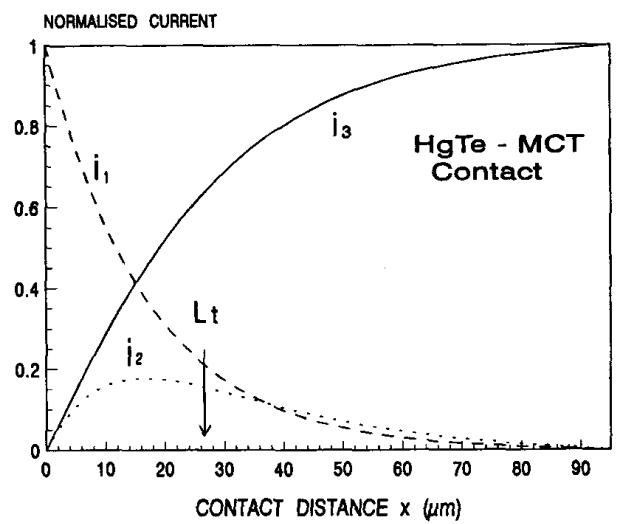

Fig. 10. Current distribution within the contact layers as a function of contact distance traversed for a $\mathrm{Au}-\mathrm{In}$ contact on a $\mathrm{HgTe} / \mathrm{HgCdTe}$ heterojunction structure. $L_{t}$ marks the value of the contact transfer length.

TABLE II

TLTLM CONTACT PARAMETERS FOR Au-In/HgTe/HgCdTe

\begin{tabular}{|c|c|c|c|c|c|c|c|}
\hline \multicolumn{8}{|c|}{ Experimental values for $\mathrm{Rc}, \operatorname{Re}$ and $\rho_{\mathrm{c}}: 88.1 \mathrm{\Omega}, 0.75 \Omega$ and $14.6 \times 10^{-4} \Omega . \mathrm{cm}^{2}$} \\
\hline \multicolumn{5}{|c|}{ TLTLM Contact Parameters: } & \multirow[b]{2}{*}{$(1-f)$} & \multicolumn{2}{|c|}{ Calculated $R c$ and $R e$ values } \\
\hline $\mathbf{R f}$ & Rsu & Rsa & $\rho_{\mathrm{ca}}$ & $\rho_{\mathrm{cu}}$ & & $\mathbf{R c}$ & $\operatorname{Re}$ \\
\hline$\infty$ & 490 & 15.0 & 0.4 & 14.02 & 0 & 88.1 & 0.75 \\
\hline$\infty$ & 500 & 15.0 & 0.6 & 13.60 & 0 & 88.1 & 0.75 \\
\hline
\end{tabular}

Resistance values in $\mathrm{\Omega}$, sheet resistance in $\mathrm{\Omega} / \mathrm{sq}$ and $\rho_{\mathrm{c}}$ values are $\times 10^{-4} \mathrm{\Omega} . \mathrm{cm}^{2}$

is only slightly modified from the $R s h$ value (490-500 $\Omega /$ sq), despite the presence of a low sheet resistance layer under the contact $(R s a=15 \Omega / \mathrm{sq})$. For the Au-Ge-Ni contact, Rsk is $100 \Omega / \mathrm{sq}$, which is a significant reduction on the $R s h$ value of $284 \Omega / \mathrm{sq}$.

\section{E. Interpretation of Standard TLM Parameters}

1) Sheet Resistance Parameter Rsk: For the example given in Table I, the standard TLM gives a value of $R s k$ of $100 \Omega / \mathrm{sq}$ for this contact although no single layer of this value is present under the contact. The original epitaxial layer sheet resistance $R s h$, was measured as $284 \Omega /$ sq. The TLTLM analysis shows that standard TLM parameter Rsk is the result of a combination of the respective sheet resistances of the alloyed $(R s a)$ and unalloyed ( $R s u$ ) layers. The exact combination depends on the respective current flows in the two layers and thus depends on $\rho_{c a}$ and $\rho_{c u}$ as well. The influence of the sheet resistance of the alloyed layer Rsa will increase as the proportion of contact current in this layer increases.

A more detailed analysis of the magnitude of $R s k$ may be made using the TLTLM network. By assigning values to the TLTLM parameters, the corresponding values of $R c$ and $R e$ can be calculated. Then, on using the standard TLM equations, the equivalent TLM parameters of $\rho_{c}$ and $R s k$ are found. Thus the effect of the various TLTLM parameters on Rsk may be predicted. Results of such an analysis [25] show that values of $R s k$ both above and below the sheet resistance $R s h$ of the epitaxial layer outside the contact region can be obtained. This is in line with experimentally reported results where for alloyed contacts $R s k$ often differs from $R s h$. Generally $R s k$ is found to be lower than Rsh [4], [12] although measured values above the value of $R s h$ have also been reported [20], [26]. For the TLTLM to predict that $R s k>R s h$, it is necessary to acknowledge that the alloying process will reduce the epilayer thickness causing $R s u$ (the sheet resistance of the semiconductor layer under the contact after alloying) to be larger than Rsh (the sheet resistance of the semiconductor layer under the contact before the contact is alloyed). The thinner the starting epitaxial layer, the greater the increase. Hence the possibility of obtaining $R s k>R s h$ will be more likely for thin epitaxial layers.

Fig. 11(a) shows the variation of $R s k$ as a function of $\rho_{c u}$ for various values of $\rho_{c a}$ for a contact $5 \mu \mathrm{m}$ long (Rsa and $R s u$ are taken as 34.4 and $440 \Omega$ /sq., respectively). Point A represents the first set of contact parameters in Table $I$. The TLTLM also shows that the calculated values of $R s k$ have a dependency on contact length. This is particularly apparent for short contacts as shown in Fig. 11(b). The solid line in this figure shows the variation of $R s k$ as a function of contact length for the contact using the first set of TLTLM parameters from Table I. The experimentally reported value of the sheet resistance outside the contact $R s h$, is marked on the figure. The dashed line shows the $R s k$ variation when the value of $\rho_{c u}$ is increased to $4 \times 10^{-6} \Omega \cdot \mathrm{cm}^{2}$. Note that even when all the electrical parameters of the contact remain unchanged, then using the standard TLM analysis, a contact of length $3 \mu \mathrm{m}$ would have given an $R s k$ value different from that of a $5 \mu \mathrm{m}$ long contact. This influence of contact geometry on $R s k$ becomes more marked as $\rho_{c u}$ increases. Had $\rho_{c u}$ been $4 \times 10^{-6} \Omega \cdot \mathrm{cm}^{2}$ then values of $R s k$ both greater and less than $R s h(284 \Omega / \mathrm{sq})$ would have been measured for contacts of say 4 and $10 \mu \mathrm{m}$, respectively.

2) Specific Contact Resistance Parameter $\rho_{c}$ : The TLTLM also describes how the standard TLM parameter $\rho_{c}$ will vary with the TLTLM parameters and with contact length. Using the same TLTLM parameters as for Fig. 11(a), the value of $\rho_{c}$ is shown in Fig. 12(a) as a function of $\rho_{c u}$ for various values of $\rho_{c a}$. In Fig. 12(b) the dependence of $\rho_{c}$ on contact length for two values of $\rho_{c u}$ is shown. The contact of Table $\mathrm{I}$ is marked by the position $\mathrm{A}$ in both figures. Some analyses based on contact current flow through the separate potential barriers at the metal-alloy and alloy-semiconductor interface infer that the experimental $\rho_{c}$ is the sum of $\rho_{c u}$ and $\rho_{c a}$ [27]. From Table $I$ it is observed that the sum of $\rho_{c u}$ and $\rho_{c a}$ is not the same as $\rho_{c}$. This is generally the case, with equality only occurring when there is no horizontal current flow in the alloyed layer $\left(i_{2}=0\right)$.

A similar calculation for the HgTe/MCT contact of Table II shows that $\rho_{c}$ has little dependency on contact length. Using the parameters of Table II and varying the contact length from $1-200 \mu \mathrm{m}$ causes the value of $\rho_{c}$ to increase by only $2.3 \%$. This follows from the fact that since $f=1.0$, then $i_{2}$ is very small for this contact. Thus the vertical voltage drop across $\rho_{c}$ closely approximates the voltage drop across $\left(\rho_{c a}+\rho_{c u}\right)$, making $\rho_{c} \approx\left(\rho_{c a}+\rho_{c u}\right)$ irrespective of the contact length. 


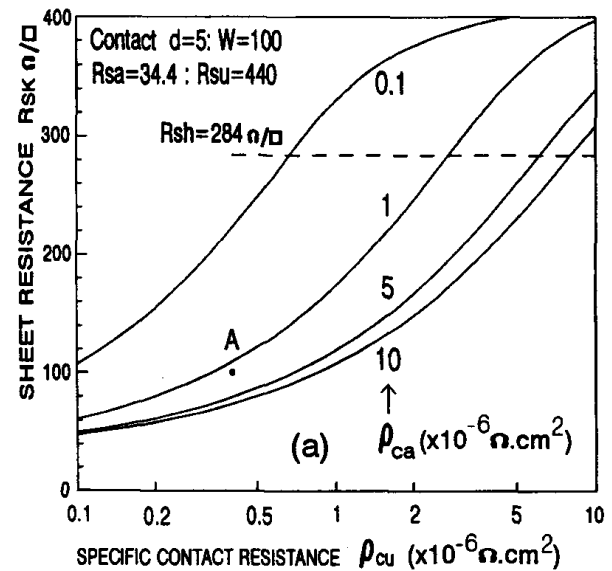

(a)

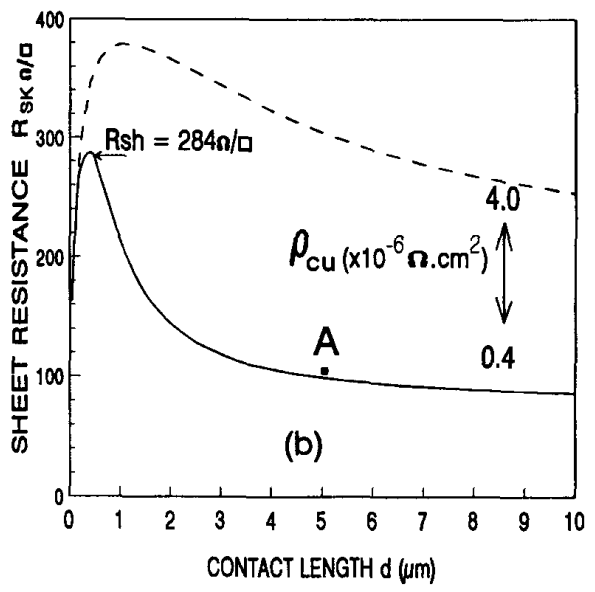

(b)

Fig. 11. Variation of standard TLM parameter $R s k$ with (a) $\rho_{c a}$ and $\rho_{c u}$ and (b) contact length $d$, for a Au-Ge-Ni alloyed contact.

As noted in the previous paragraph, had $i_{2}$ equaled zero, then $\rho_{c}$ would equal $\left(\rho_{c a}+\rho_{c u}\right)$.

The contribution from one of the two interfaces may dominate the measured $\rho_{c}$. The TLTLM model predicts that for the contact in Table II, most of the relatively large value of $\rho_{c}$ measured is due to the HgTe-HgCdTe interface $\left(\rho_{c u}\right)$. For the Au-Ge-Ni contact summarized in Table I, the major contribution to the standard TLM parameter $\rho_{c}$, comes from $\rho_{c a}$, although the contribution from the two interfaces is not nearly as unequal as for the heterojunction contact of Table II. It was pointed out in Section IV-E-1 that $R s k$ could be greater or less than $R s h$. While the calculated value of $\rho_{c}$ is generally greater than $\left(\rho_{c a}+\rho_{c u}\right)$, it is possible for it to be less as well. This occurs for contacts where $\rho_{c a}$ is smaller than $\rho_{c u}$ and where $f$ is small, i.e., a significant portion of the contact current enters the alloyed layer via $R f$ and thus by-passes the $\rho_{c u}$ interface beneath the contact. However this tendency is also counteracted by the large $\rho_{c u}$ which in turn increases both $R f$ and $f$. For example in Fig. 12(a), $\rho_{c}<\left(\rho_{c a}+\rho_{c u}\right)$ on the line where $\rho_{c a}=0.1 \times 10^{-6} \Omega \cdot \mathrm{cm}^{2}$ provided $\rho_{c u}>1 \times 10^{-6} \Omega \cdot \mathrm{cm}^{2}$.

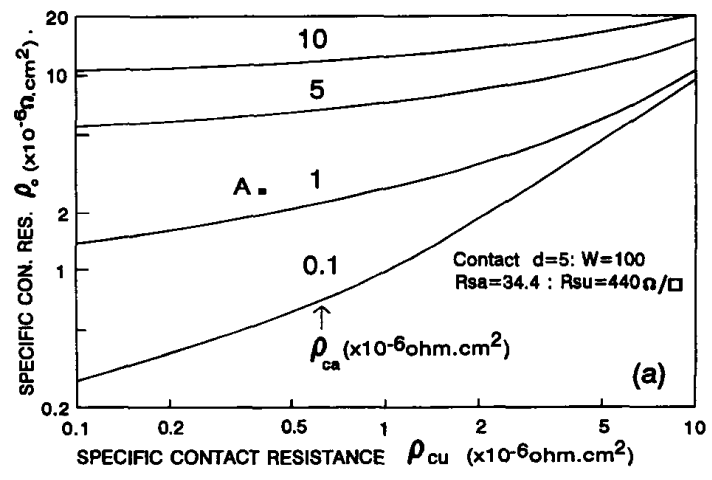

(a)

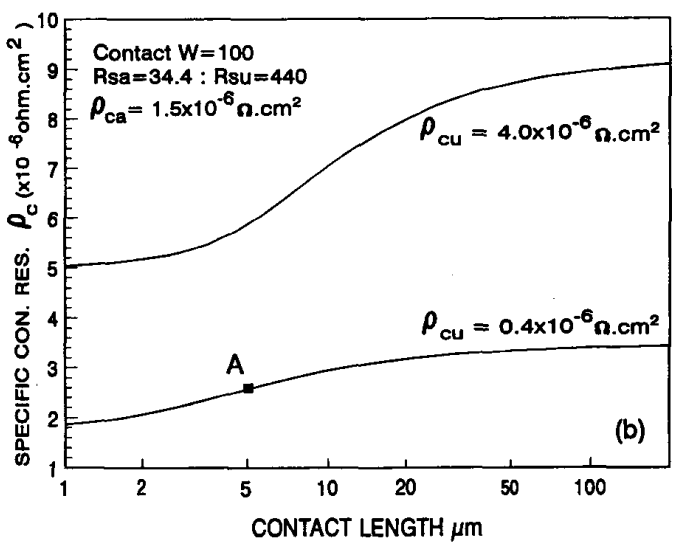

(b)

Fig. 12. Variation of standard TLM parameter $\rho_{c}$ with (a) $\rho_{c a}$ and $\rho_{c u}$, and (b) contact length $d$, for a Au-Ge-Ni alloyed contact.

The results of Figs. 11 and 12 indicate that in using the standard TLM to analyze alloyed ohmic contacts, the contact length can affect the values of both $R s k$ and $\rho_{c}$ even when the contacts are electrically identical.

\section{F. Contact End Resistance}

In the standard TLM, the contact end resistance $R e$ is given by (1) as $V_{C D} / i_{0}$ where, with reference to Fig. $1, V_{C D}$ is the voltage drop across the contact interface at $C D$ at the far end of the contact. The current $i_{0}$ is injected into the opposite end of the contact at node $B$. From Fig. 13(a), the voltage $V_{C D}$ is measured by assuming that the third metal contact (contact 3) adjacent to contact 2 is at the same potential as node $C$, and thus $V_{C D}$ is equated to the voltage between contacts 2 and 3 [12].

In the TLTLM, the contact end resistance is similarly defined as the ratio of the vertical voltage drop across the metal-semiconductor interface to the injected current $i_{0}$ (see Fig. 5 and (5)). However in this model, the existence of two interfaces $\rho_{c a}$ and $\rho_{c u}$ suggests that two end resistance terms can be defined. The first end resistance term is the one that has been defined in (5) and considered up to this point. The second end resistance term $R^{\prime \prime} e$ is defined as $V_{Y Z} / i_{0}$ (Fig. 13(b)), and following the procedure used for deriving $\operatorname{Re}$ (A17), it may 


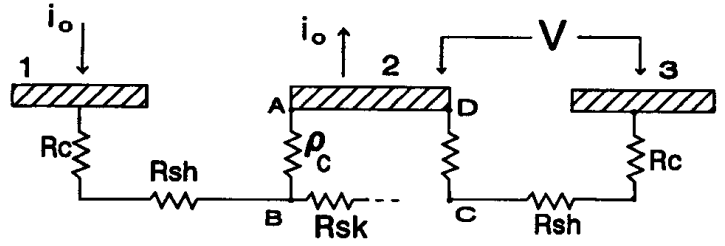

(a)

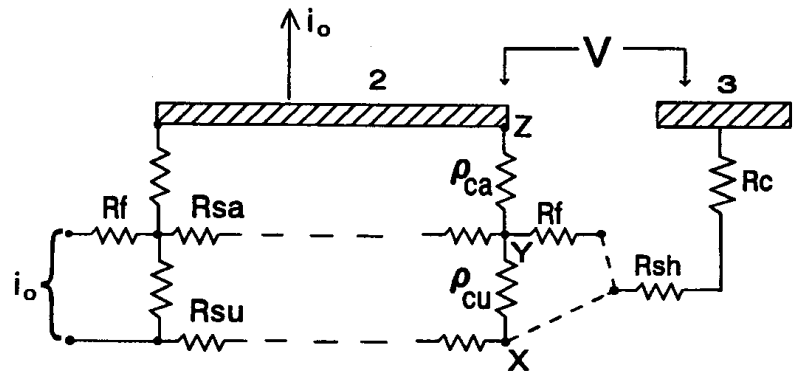

(b)

Fig. 13. (a) Standard TLM network for a three-contact test pattern showing the method for measuring $R e$, and (b) the same measurement on the TLTLM network illustrating how the potential at nodes $X$ and $Y$ of contact 2 can affect $V$ and hence $R e$.

be shown that

$$
\begin{aligned}
R^{\prime \prime} e= & \left\{\frac{R s a}{\rho_{c u} \cdot\left(b^{2}-a^{2}\right) \cdot w}\right\} \cdot\left\{\frac{\rho_{c u} \cdot b^{2}(1-f)-R s u}{b \cdot \sinh (b d)}\right. \\
& \left.-\frac{\rho_{c u} \cdot a^{2}(1-f)-R s u}{a \cdot \sinh (a d)}\right\} .
\end{aligned}
$$

If the electrical model for the standard TLM is followed, the potential on contact 3 of the test pattern is taken as the same as the potential on node $X$ (equivalent to node $C$ in the standard TLM). However as Fig. 13(b) shows, in the TLTLM, the alloyed layer just beneath the contact (sheet resistance $R s a$ ) is electrically connected (from node $Y$ ) to the semiconductor material outside the contact by a resistance $R f$ - equivalent to the $R f$ present at the leading edge of the contact (4). Thus the potential on contact 3 will probably have a value somewhere between the potential of nodes $X$ and $Y$. Exactly where between $R e$ and $R^{\prime \prime} e$ the iend resistance will lie, will also depend on such factors as the magnitudes of $R f$ and $R s h$ (since current now flows between $X$ and $Y$ via $R f$ and $R s h$, as well as through $\rho_{c u}$ ), the distance between contacts 2 and 3 and the thickness of the active semiconductor layer. Note that for the $\mathrm{HgTe} / \mathrm{MCT}$ contact that since $f=1.0(R f \rightarrow \infty)$, then no $R^{\prime \prime} e$ term exists.

It is useful to compare the ratio of $R e: R^{\prime \prime} e$ and thus establish under what conditions $R e$ differs from $R^{\prime \prime} e$. This in turn would indicate if a significant error could arise by taking $R e$ as the end resistance rather than some average of $R e$ and $R^{\prime \prime} e$. The ratio $R e: R^{\prime \prime} e$ is graphed in Fig. 14(a) as a function of $\rho_{c u}$ for several values of $\rho_{c a}$. The other contact parameters are the same as for Fig. 13. In Fig. 14(b), the same ratio is graphed as a function of contact length. Depending on the TLTLM parameters, the length can influence the value of $R e: R^{\prime \prime} e$.

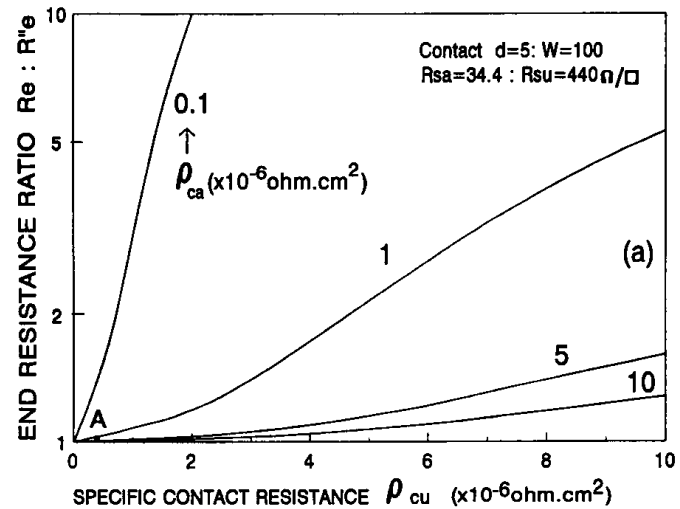

(a)

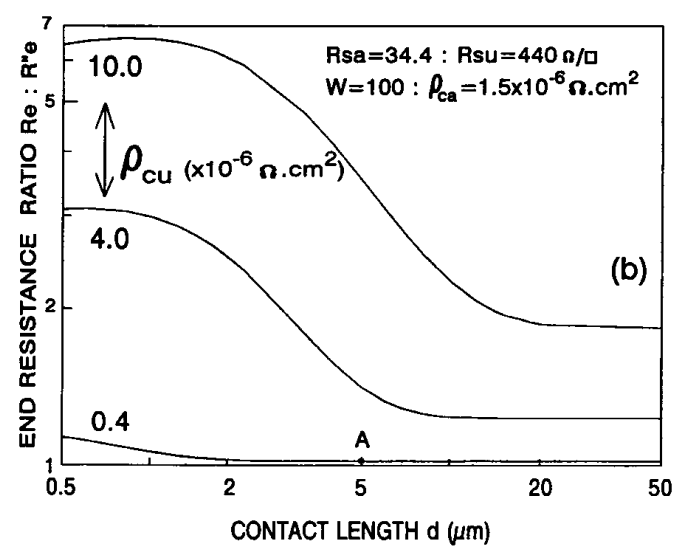

(b)

Fig. 14. Variation of the ratio of $R e: R^{\prime \prime} e$ with (a) $\rho_{c a}$ and $\rho_{c u}$, and (b) contact length $d$, for a Au-Ge-Ni alloyed contact.

Generally any variations in $R e: R^{\prime \prime} e$ only occur for lengths up to $\sim 20 \mu \mathrm{m}$. Beyond this $R e: R^{\prime \prime} e$ tends to a constant value.

\section{CONCLUSION}

A new electrical model based on TLM principles has been proposed in order to more accurately model the known physical structure of an alloyed ohmic contact. The model allows the alloyed layer beneath the contact to be characterized by its own sheet resistance. It is also characterized by two independent specific contact resistances-one for the metal:alloyed layer interface and the second for the alloyed:semiconductor layer interface. In addition, the TLTLM model can account for the current crowding due to current directly entering the sidewall of the alloyed layer at the leading edge of the contact. The model is applicable not only to alloyed ohmic contacts, but to other contact structures such as nonalloyed $n^{+} / n$ and heterojunction contacts. Examples of deriving a set of TLTLM parameters from the experimental values of $R c$ and $R e$ of an alloyed $\mathrm{GaAs}$ contact and a heterojunction $\mathrm{HgTe} / \mathrm{HgCdTe}$ are given. The results show that quite plausible values of the TLTLM parameters are derived when the constraint of obtaining agreement with the measured $R c$ and $R e$ is applied. 


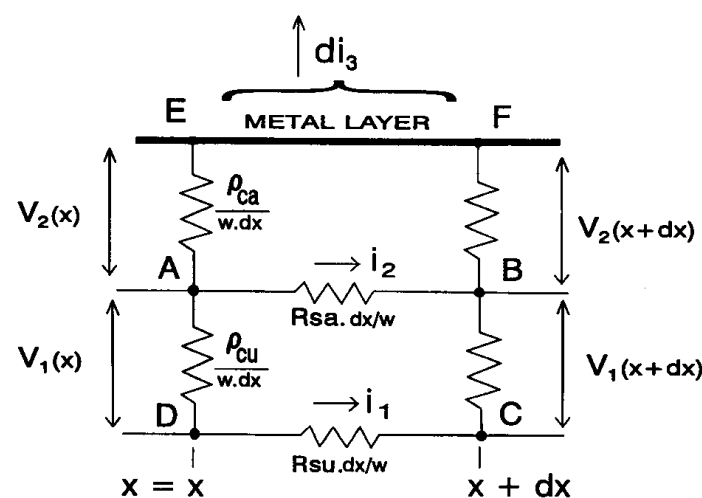

Fig. 15. The TLTLM network for deriving (A1)-(A4).

The model also demonstrates the way in which the standard TLM parameters $R s k$ and $\rho_{c}$ can be influenced by the contact length, as well as the sheet resistance and the interfaces defined by using the TLTLM network. It highlights inaccuracies that may arise when deriving standard TLM parameters $\rho_{c}$ and $R s k$ from test patterns using short contact lengths.

New test patterns would clearly be useful in order to directly determine the specific contact resistances of the interfaces $\rho_{c a}$ and $\rho_{c u}$ and possibly $R s a$. This would further clarify the contributions to the resistance of the planar ohmic contact.

\section{APPENDIX}

\section{A. Derivation of Current Flow}

Referring to Fig. 15, and summing the potentials around the loop DABC, then

$$
\begin{aligned}
& \begin{aligned}
& V_{1}(x)+i_{2}(x) \cdot R s a \cdot d x / w \\
&= V_{1}(x+d x)+i_{1}(x) \cdot R s u \cdot d x / w \text { or }
\end{aligned} \\
& d V_{1}(x) / d x=\left(i_{2}(x) \cdot R s a-i_{1}(x) \cdot R s u\right) / w .
\end{aligned}
$$

Likewise from loop AEFB

$$
d V_{2}(x) / d x=-i_{2}(x) \cdot R s a / w .
$$

Across the resistor $\operatorname{AD}\left(\rho_{c u} /(w \cdot d x)\right)$

$$
V_{1}(x)=-\left(\rho_{c u} / w\right) \cdot d i_{1} / d x
$$

and across the resistor $\operatorname{AE}\left(\rho_{c a} /(w \cdot d x)\right)$

$$
\begin{aligned}
V_{2}(x) & =\left(\rho_{c a} / w\right) \cdot d i_{3} / d x \text { or } \\
d V_{2}(x) / d x & =\left(\rho_{c a} / w\right) \cdot d^{2} i_{3} / d x^{2} .
\end{aligned}
$$

(Note: the signs in (A3) and (A4) reflect the fact that $i_{1}(x)$ decreases as $x$ increases while $i_{3}(x)$ increases as $x$ increases). Equating (A2) and (A4) and using primes to denote the differentials $\left(i_{3}^{\prime \prime}=d^{2} i_{3} / d x^{2}\right)$

$$
\rho_{c a} \cdot i_{3}^{\prime \prime}=-i_{2} \cdot R s a=-R s a \cdot\left(i_{0}-i_{1}-i_{3}\right)
$$

as $i_{0}=i_{1}+i_{2}+i_{3}$.

Equating (A1) and the differential of (A3)

$$
\rho_{c u} \cdot i_{1}^{\prime \prime}=\left(i_{1} \cdot R s u-i_{2} \cdot R s a\right) .
$$

Since $i_{0}=i_{1}+i_{2}+i_{3}$, then on substituting for $i_{2}$ and rearranging

$$
i_{3} \cdot R s a=i_{0} \cdot R s a+\rho_{c u} \cdot i_{1}^{\prime \prime}-i_{1} \cdot R s^{\prime}
$$

where $R s^{\prime}=R s a+R s u$. On differentiating twice

$$
i_{3}^{\prime \prime}=\left(\rho_{c u} \cdot i_{1}^{\prime \prime \prime}-i_{1}^{\prime \prime} \cdot R s^{\prime}\right) / R s a .
$$

Substituting (A8) into (A5) for $i_{3}^{\prime \prime}$ and substituting (A7) into (A5) for $i_{3}$, then (A5) becomes a fourth-order differential equation in $i_{1}$

$$
\begin{aligned}
i_{1}^{\prime \prime \prime \prime}- & i_{1}^{\prime \prime}\left(R s a / \rho_{c a}+R s^{\prime} / \rho_{c u}\right) \\
& +i_{1} \cdot R s a \cdot R s u /\left(\rho_{c u} \cdot \rho_{c a}\right)=0 .
\end{aligned}
$$

Equation (A9) may be solved by rewritng and factorizing

$$
\left(D^{2}-A\right)\left(D^{2}-B\right) \cdot i_{1}=0
$$

where the operator $D \equiv d / d x$ and $A+B=\left(R s a / \rho_{c a}+\right.$ $\left.R s^{\prime} / \rho_{c u}\right)$ and $A \cdot B=R s a \cdot R s u /\left(\rho_{c u} \cdot \rho_{c a}\right)$.

The general solution is obtained in the normal way by summing the complementary function and the particular integral. Thus

$$
i_{1}(x)=\left(C_{1} e^{a x}+C_{2} e^{-a x}\right) /\left(a^{2}-b^{2}\right)+C_{3} e^{b x}+C_{4} e^{-b x}
$$

where $a=A^{1 / 2}$ and $b=B^{1 / 2}$.The first term in (A10) represents the particular integral and the last two terms, the complementary function. The constants $a$ and $b$ are given by

$$
\begin{aligned}
a & \left.=\sqrt{[c}-\left(c^{2}-4 \cdot R s u \cdot R s a /\left(\rho_{c u} \cdot \rho_{c a}\right)\right)^{1 / 2} / 2\right] \\
b & \left.=\sqrt{[} c+\left(c^{2}-4 \cdot R s u \cdot R s a /\left(\rho_{c u} \cdot \rho_{c a}\right)\right)^{1 / 2} / 2\right]
\end{aligned}
$$

where $c=\left[R s^{\prime} / \rho_{c u}+R s a / \rho_{c a}\right]$.

The constants $C_{1}-C_{4}$ are determined from the boundary conditions

$$
\begin{aligned}
& \text { At } x=0: i_{1}(0)=f . i_{0} \quad i_{2}(0)=(1-f) \cdot i_{0} \quad i_{3}(0)=0 \\
& \text { Atx }=d: i_{1}(d)=0 \quad i_{2}(d)=0 \quad i_{3}(d)=i_{0} .
\end{aligned}
$$

Thus it may be shown that

$$
\begin{aligned}
C_{1}= & i_{0} \cdot\left[f \cdot\left(R s u-\rho_{c u} \cdot b^{2}\right)\right. \\
& -R s a(1-f)] /\left[\rho_{c u}\left(1-e^{2 a d}\right)\right] \\
C_{2}= & i_{0} \cdot\left[f \cdot\left(R s u-\rho_{c u} \cdot b^{2}\right)\right. \\
& -R s a(1-f)] /\left[\rho_{c u}\left(1-e^{-2 a d}\right)\right] \\
C_{3}= & i_{0} \cdot\left[f \cdot\left(R s u-\rho_{c u} \cdot a^{2}\right)\right. \\
& -R s a(1-f)] /\left[\rho_{c u}\left(b^{2}-a^{2}\right) \cdot\left(1-e^{2 b d}\right)\right] \\
C_{4}= & i_{0} \cdot\left[f \cdot\left(R s u-\rho_{c u} \cdot a^{2}\right)\right. \\
& -R s a(1-f)] /\left[\rho_{c u}\left(b^{2}-a^{2}\right) \cdot\left(1-e^{-2 b d}\right)\right] .
\end{aligned}
$$

Thus on substitution into (A10) and simplifying, the equation for $i_{1}(x)$ becomes

$$
\begin{aligned}
i_{1}(x)= & \left\{\frac{i_{0}}{\rho_{c u} \cdot\left(b^{2}-a^{2}\right)}\right\} \cdot\left\{\frac{P \cdot \sinh b(d-x)}{\sinh (b d)}\right. \\
& \left.-\frac{Q \cdot \sinh a(d-x)}{\sinh (a d)}\right\}
\end{aligned}
$$




$$
f=\left\{\frac{N+R s a \cdot[b \cdot \tanh (a d)-a \cdot \tanh (b d)]}{N+\left(R s a+R s u-\rho_{c u} \cdot a^{2}\right) \cdot b \cdot \tanh (a d)-\left(R s a+R s u-\rho_{c u} \cdot b^{2}\right) \cdot a \cdot \tanh (b d)}\right\}
$$

where $P=f \cdot\left(R s u-\rho_{c u} \cdot a^{2}\right)-(1-f) \cdot R s a$ and $Q=f \cdot\left(R s u-\rho_{c u} \cdot b^{2}\right)-(1-f) \cdot R s a$.

The equation for $i_{2}(x)$ may be found on substitution into (A6) to be

$$
\begin{aligned}
i_{2}(x)= & \left\{\frac{i_{0}}{R s a \cdot \rho_{c u} \cdot\left(b^{2}-a^{2}\right)}\right\} \\
& \cdot\left\{\frac{P \cdot\left(R s u-\rho_{c u} \cdot b^{2}\right) \cdot \sinh b(d-x)}{\sinh (b d)}\right. \\
& \left.-\frac{Q \cdot\left(R s u-\rho_{c u} \cdot a^{2}\right) \cdot \sinh a(d-x)}{\sinh (a d)}\right\} .
\end{aligned}
$$

Likewise, expressions for the potentials $V_{1}(x)$ and $V_{2}(x)$ may be calculated from (A3) and (A2), respectively,

$$
\begin{aligned}
V_{1}(x)= & \left\{\frac{i_{0}}{\left(b^{2}-a^{2}\right) \cdot w}\right\} \cdot\left\{\frac{P \cdot b \cdot \cosh b(d-x)}{\sinh (b d)}\right. \\
& \left.-\frac{Q \cdot a \cdot \cosh a(d-x)}{\sinh (a d)}\right\} \\
V_{2}(x)= & \left\{\frac{i_{0}}{\rho_{c u} \cdot\left(b^{2}-a^{2}\right) \cdot w}\right\} \\
& \cdot\left\{\frac{P \cdot\left(R s u-\rho_{c u} \cdot b^{2}\right) \cdot \cosh b(d-x)}{b \cdot \sinh (b d)}\right. \\
& \left.-\frac{Q \cdot\left(R s u-\rho_{c u} \cdot a^{2}\right) \cdot \cosh a(d-x)}{a \cdot \sinh (a d)}\right\} .
\end{aligned}
$$

\section{B. Derivation of $R c$ and $R e$}

The total potential $V(x)$ across both interfaces $\rho_{c a}$ and $\rho_{c u}$ is found by summing $V_{1}(x)$ and $V_{2}(x)$

$$
\begin{aligned}
V(x)= & \left\{\frac{i_{0} \cdot R s u}{\rho_{c u} \cdot\left(b^{2}-a^{2}\right) \cdot w}\right\} \cdot\left\{\frac{P \cdot \cosh b(d-x)}{b \cdot \sinh (b d)}\right. \\
& \left.-\frac{Q \cdot \cosh a(d-x)}{a \cdot \sinh (a d)}\right\} .
\end{aligned}
$$

In Fig. 16, points 1 and 2 lie on an equipotential at the front edge of the contact. The device current enters the contact at this equipotential which is $V(0)$ volts above the potential of the metal layer. Thus $R c=V(0) / i_{0}$. On taking $x=0$ and dividing by $i_{0}$ then $R c$ is found to be

$$
\begin{aligned}
R c= & \left\{\frac{R s u}{\rho_{c u} \cdot\left(b^{2}-a^{2}\right) \cdot w}\right\} \cdot\left\{\frac{P}{b \cdot \tanh (b d)}\right. \\
& \left.-\frac{Q}{a \cdot \tanh (a d)}\right\} .
\end{aligned}
$$

The contact end resistance is calculated from the definition $R e=V_{X Z} / i_{0}$ (see Fig. 13(b)). Since $V_{X Z}=V(d)$ then using (A15)

$$
\begin{aligned}
R e= & \left\{\frac{R s u}{\rho_{c u} \cdot\left(b^{2}-a^{2}\right) \cdot w}\right\} \cdot\left\{\frac{P}{b \cdot \sinh (b d)}\right. \\
& \left.-\frac{Q}{a \cdot \sinh (a d)}\right\}
\end{aligned}
$$

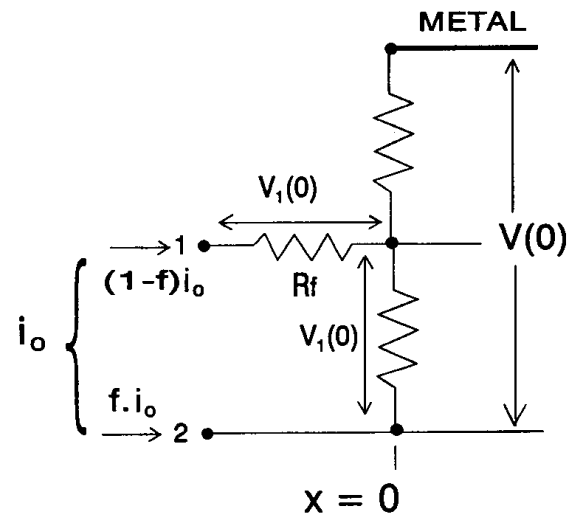

Fig. 16. Potential drops at the contact front edge.

\section{Derivation of Current Division Factor $f$}

The current division factor $f$ can be determined from (A18) which is derived from Fig. 16

$$
V_{1}(0)=(1-f) \cdot i_{0} \cdot R f .
$$

On substituting for $V_{1}(0)$ using (A13) and writing $x=0$ and rearranging (see (A19) at the top of this page), where $N=R f \cdot w \cdot\left(b^{2}-a^{2}\right) \cdot \tanh (b d) \cdot \tanh (a d)$.

\section{REFERENCES}

[1] R. Zuleeg, P. E. Frieberthauser, J. M. Stephens, and S. H. Watanabe, "Al-Ge ohmic contacts to $n$-type GaAs," IEEE Electron. Device Lett., vol. EDL-7, no. 11, pp. 603-604, 1986.

[2] M. Murakami, A. Otsuki, K. Tanahashi, and H. J. Takata, "Recent progress of alloyed ohmic contacts to GaAs compounds," in MRS Symp. Proc., vol. 260, 1992, pp. 457-467.

[3] W. Kellner, "Planar ohmic contacts to $N$-type GaAs: Determination of contact parameters using the transmission line model," Siemens Forsch.u. Entwickl.-Ber, vol. 4, no. 3, pp. 137-140, 1975.

[4] M. Ogawa, "Alloying behavior of Ni/Au-Ge films on GaAs," J. Appl. Phys., vol. 51, no. 1, pp 406-412, 1980.

[5] H. Berger, "Models for contacts to planar devices," Solid-State Electron., vol. 15, pp. 145-158, 1972.

[6] G. K. Reeves and H. B. Harrison, "Determination of contact parameters of interconnecting layers in VLSI circuits," IEEE Trans. Electron Devices, vol. ED-33, no. 3, pp. 328-334, 1986.

[7] D. B. Scott, W. R. Hunter, and H. Shichijo, "A transmission line model for silicided diffusions: Impact on the performance of VLSI circuits," IEEE Trans. Electron Devices, vol. ED-29, no.4, pp. 651-661, 1982.

[8] M. D. Feuer, "Two-layer model for source resistance in selectively doped heterojunction transistors," IEEE Trans. Electron Devices, vol. ED-32, no. 1 , pp. 7-11, 1985.

[9] J. M. Pimbley, "Dual-level transmission line model for current flow in metal-semiconductor contacts," IEEE Trans. Electron Devices, vol. ED-33, no. 11, pp. 1795-1800, 1986.

[10] W. Shockley, "Research and investigation of inverse epitaxial UHF power transistors," Air Force Atomic Laboratory, Wright-Patterson Air Force Base, Rep. No. AL-TDR-64-207, Sept. 1964.

[11] R. H. Cox and H. Strack, "Ohmic contacts for GaAs devices," SolidState Electron., vol. 10, pp. 1213-1218, 1967.

[12] G. K. Reeves and H. B. Harrison, "Obtaining the specific contact resistance from transmission line model measurements," IEEE Electron Device Lett., vol. EDL-3, no. 5, pp. 111-113, 1982. 
[13] S. Proctor and L. Linholm, "A direct measurement of interfacial contact resistance," IEEE Electron Dev. Lett., vol. EDL-3, no. 10, pp. 294-296, 1982.

[14] H. B. Harrison and G. K. Reeves, "Assessing ohmic contacts," in MRS Symp. Proc., vol. 260, 1992, pp. 31-42.

[15] N. Braslau, "Alloyed ohmic contacts to GaAs," J. Vac. Sci. Technol., vol. 19 , no. 3, pp. 803-807, 1981.

[16] T. S. Kuan, P. E. Batson, T. N. Jackson, H. Rupprecht, and E. L. Wilkie "Electron microscope studies of an alloyed $\mathrm{Au} / \mathrm{Ni} / \mathrm{Au}-\mathrm{Ge}$ ohmic contact to GaAs," J. Appl. Phys., vol. 54, no. 12, pp. 6952-6957, 1983.

[17] M. Murakami, K. D. Childs, J. M. Baker, and A. Callegari, "Microstructure studies of AuNiGe ohmic contacts to $n$-type GaAs," J. Vac. Sci. Technol., vol. B4, no. 4, pp. 903-911, 1986.

[18] A. Callegari, M. Murakami, J. M. Baker, Y. C. Shih, and D. Lacey, "Temperature stability of AuGeNi ohmic contacts to GaAs," in Proc. 17th Eur. Sol. Stat. Dev. Res. Conf., ESSDERC 87, 1988, pp. 835-838.

[19] H. Goronkin, S. Tehrani, T. Remmel, P. Fejes, and K. J. Johnson, "Ohmic contact penetration and encroachment in GaAs/AlGaAs and GaAs FET's," IEEE Trans. Electron Devices, vol. 36, no. 2, pp. 281-288, 1989.

[20] H. G. Henry, "Characterization of alloyed AuGe/Ni/Au ohmic contacts to $n$-doped GaAs by measurement of transfer length and under the contact sheet resistance," IEEE Trans. Electron Devices, vol. 36, no. 7, pp. 1390-1393, 1989.

[21] P. A. Barnes and A. Y. Cho, "Nonalloyed ohmic contacts to $n-G a A s$ by molecular beam epitaxy," Appl. Phys. Lett., vol. 33, no. 7, pp. 651-653, 1978.

[22] K. Shenai, "Very low resistance nonalloyed ohmic contacts to Sn-doped molecular-beam epitaxial GaAs," IEEE Trans. Electron Devices, vol. ED-34, no. 8, pp. 1642-1649, 1987.

[23] K. Shenai and R. W. Dutton, "Current transport mechanisms in atomically abrupt metal-semiconductor interfaces," IEEE Trans. Electron Devices, vol. ED-35, no. 4, pp. 468-482, 1988.

[24] P. W. Leech and G. K. Reeves, "Properties of HgTe as a contact layer to $\mathrm{n}-\mathrm{Hg}_{1-x} \mathrm{Cd}_{x} \mathrm{Te}$," Semicon. Sci. and Technol., vol. 8, no. 12, pp. 2097-2100, 1993.

[25] G. K. Reeves, P. W. Leech, and H. B. Harrison, "Understanding the sheet resistance parameter for alloyed ohmic contacts using a transmission line model," Solid-State Electron., vol. 38, no. 4, pp. 745-751, 1995.
[26] H. P. Kattelus, J. L. Tandon, and M-A. Nicolet, "Characterization of reacted ohmic contacts to GaAs," Solid-State Electron., vol. 29, no. 9, pp. 903-905, 1986.

[27] W. Dingfen, W. Dening, and K. Heime, "An improved model to explain ohmic contact resistance of $n$-GaAs and other semiconductors," SolidState Electron., vol. 29, no. 5, pp. 489-494, 1986.

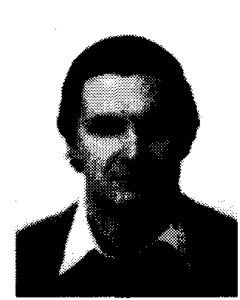

Geoffrey K. Reeves received the Ph.D. degree in solid-state physics from Monash University, Melbourne, Australia, in 1970.

He subsequently held several industrial research and development positions, before joining the Research Laboratories of Telecom Australia. Here he undertook research on compound semiconductor materials and optoelectronic devices, including work on the electrical characterization of ohmic contacts. In 1988, he took up a research position at the Royal Melbourne Institute of Technology, where he continued research on semiconductor materials and the development of fabrication technologies for Microwave Integrated Circuits.

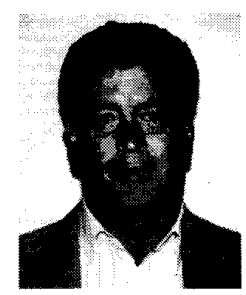

H. Barry Harrison received the B.E. degree in electrical engineering (honors) and the Diploma of Education from the University of Melbourne, Australia, in 1965 and 1967, respectively. He received a graduate degree in communication engineering in the Netherlands in 1972 .

He has held various academic and industrial positions around the world, such as at Philips Research Laboratory in the Netherlands, and the IBM T. J. Watson Research Laboratories and Bell AT\&T in the United States. He served as a Professor at the University of Edinburgh, Scotland and Leuven in Belgium. He is currently Deputy Dean of the Faculty of Science and Technology, and Head of the School of Microelectronic Engineering at Griffith University. 